\title{
STUDY OF FERRO RESONANCE USING GENERALIZED MODELS OF PASSIVE NONLINEAR ELEMENTS
}

\author{
Shavkat Begmatov ${ }^{1}$, Saidakhon Dusmukhamedova ${ }^{1}$, Kholzhon Holbutaeva ${ }^{1}$ \\ ${ }^{1}$ Tashkent state technical university, University street №2, Tashkent, 100095, Uzbekistan
}

\begin{abstract}
The requirements for reliable power supply in the power supply system determines the need to study the modes of operation of electrical systems with the problem of damage to power lines due to ferroresonance phenomena. In this regard, an effective method is proposed for the analysis of ferroresonance arising in electrical lines between inductors and capacities using generalized models of passive nonlinear elements. The method of analysis makes it possible to obtain qualitatively new and sufficiently accurate analytical expressions for calculating the electromagnetic modes of ferroresonance.
\end{abstract}

\section{Introduction}

Requirements for reliable power supply to consumers determine the need to study the operating modes of electrical systems that lead to damage to electrical equipment and disruption of power supply. One of the important reasons for the occurrence of such modes are ferroresonance phenomena (FP). It is argued that the most frequent causes of damage to electrical equipment are ferroresonance between the network capacity and the inductance of the power step-down transformer [1].

In the electric power industry, FP occurs in electrical networks during the formation of circuits with electromagnetic transformers (EMT) and network capacities, characterized by long-term overvoltages and current overloads of voltage transformer windings due to abrupt saturation of the transformer magnetic circuits. Overvoltages arising in this case are also dangerous for measuring equipment and protection means of power equipment. Therefore, the problem of analyzing FP in electrical networks requires the creation of qualitatively new mathematical models and analytical equations, which allow obtaining sufficiently accurate analytical expressions for calculating the electromagnetic modes of ferroresonance.

\subsection{Generalized models of passive nonlinear elements}

Passive nonlinear elements (PNE) - nonlinear inductance, capacitance and resistive resistance are widely used in electrical systems. The creation of their generalized models and the methodology for determining their equivalent parameters have theoretical and practical implications.

It is known that the dependence of the magnetic induction $\mathrm{b}$ on the field strength $\mathrm{h}$, taking into account the surface effect and the dynamic properties of the substance, is expressed by the equation [2]: $b=F_{1}\left(h, \frac{d h}{d t}, \ldots, \frac{d b}{d t}, \frac{d^{2} b}{d t^{2}}, \ldots\right)$.

In practice, the dependence of the current $i$ on the flux linkage $\psi$ in a nonlinear inductance (NI) is often used. In this case, if we do not take into account the derivatives of higher order, then formula (1) will take the following form [4]:

$$
i=F_{2}\left(\psi, \psi^{n} ; \frac{d \psi}{d t}, \frac{d^{2} \psi}{d t^{2}} \ldots\right)
$$

Equation (2) in the general case describes the equivalent circuit of the NI, which is its generalized model (Fig. 1).

If we assume that the equivalent parameters Ls, $\mathrm{C}_{3}$, and gэ in this circuit are constant, then we obtain $i=C_{\supset} \frac{d^{2} \psi}{d t^{2}}+g_{\supset} \frac{d \psi}{d t}+a \psi+b \psi^{n}+\frac{\psi}{L_{S}}$

where is the approximation of the Weber-ampere characteristic of the NI, obtained on the basis of the magnetization curve $\mathrm{B}=\mathrm{f}(\mathrm{H}), \mathrm{Cэ}$ is the equivalent electromagnetic capacitance of the NI, is the equivalent active conductivity of the NI; Ls is the leakage inductance of NI.

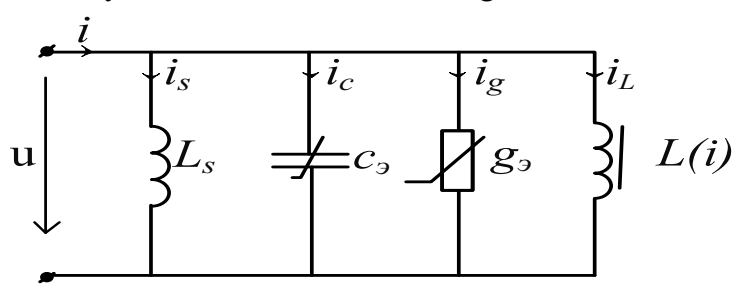

Fig. 1. Generalized NI model

Let us assume that the voltage across the inductor is described by the equation $u=U_{m} \operatorname{Cos} \omega t$. Then 
$\left\{\begin{array}{l}i_{1}=\frac{\psi}{L_{S}}=\text { аж; где } a=\frac{1}{L_{S}} ; \\ \psi=\frac{U_{m}}{\omega} \sin \omega t=\Psi_{m} \sin \omega t ; \\ i_{C}=C_{\ni} \frac{d^{2 \psi}}{d t^{2}}=-\omega^{2} C_{\ni} \Psi_{m} \sin \omega t=-I_{C m} \sin \omega t ; \\ i_{g}=\frac{1}{R_{\ni}} \cdot \frac{d \psi}{d t}=\frac{\Psi_{m} \omega}{R_{\ni}} \cos \omega t=I_{g m} \cos \omega t .\end{array}\right.$

From (4), taking into account the adopted approximation, it follows:

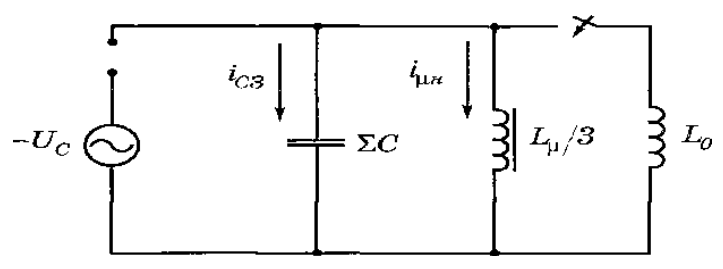

Fig. 3. Equivalent circuit of ferroresonance with EMT and network capacities

Let us apply expression (3) for the equivalent circuit of ferroresonance with EMT and network capacitances (Fig. 1.) with the assumption that the equivalent parameters $\sum g, \sum C$ and $L_{0}$ are constant and then we have

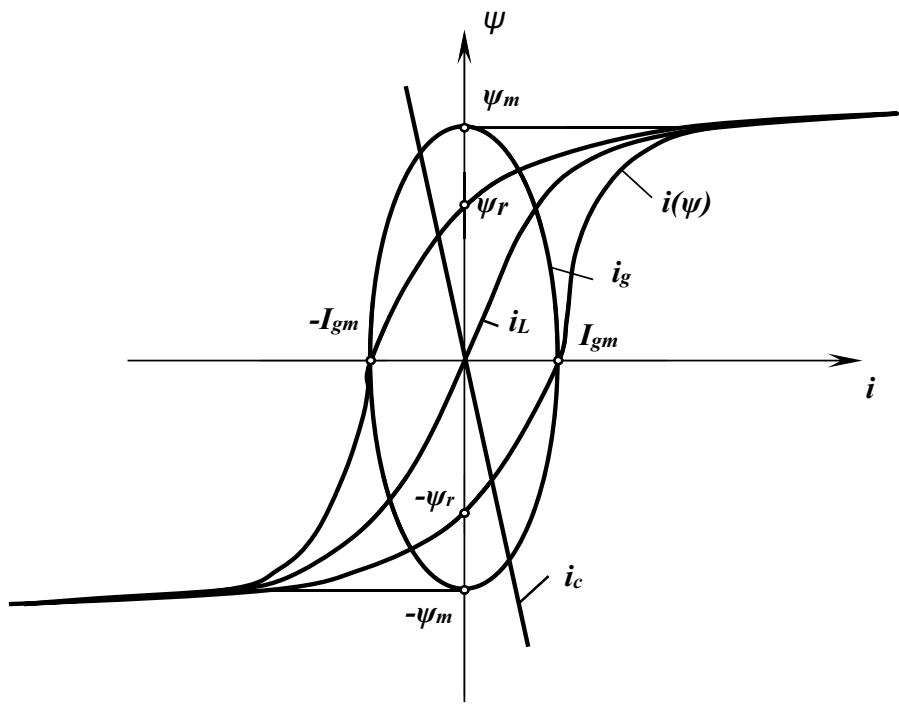

Fig. 2. Dynamic hysteresis loop NI

$\left\{\begin{array}{l}i_{c}=-\frac{I_{c m}}{\Psi_{m}} \psi ; \\ i_{g}= \pm \frac{I_{g m}}{\Psi_{m}} \sqrt{\Psi_{m}^{2}-\psi^{2}} ; \\ i_{L}{ }^{\prime}=a \psi+b \psi^{n},\end{array}\right.$

where $i_{L}^{\prime}=i_{1}+i_{L}$.

On the basis of system (5), it is possible to construct the dynamic characteristic of the NI, which is its hysteresis loop (Fig. 2) [4,5]:

Also from (4) we have

$i=\left(a-\frac{I_{c m}}{\Psi_{m}}\right) \psi+b \psi^{n} \pm \frac{I_{g m}}{\Psi_{m}} \sqrt{\Psi_{m}^{2}-\psi^{2}}$

\subsection{Ferroresonance research using generalized models of passive nonlinear elements}

Let's conduct a study of ferroresonance in the equivalent circuit with EMT and network capacities (Fig. 3) using the generalized model of nonlinear inductance (NI) (Fig. 2) [7]:

$$
i=\sum C \frac{d^{2} \psi}{d t^{2}}+\sum g \frac{d \psi}{d t}+a \psi+b \psi^{n}+\frac{\psi}{L_{0}}
$$

Where $\sum C$ - total electromagnetic capacitance, $i_{\mu H}=a \psi+b \psi^{n}$ - approximation of the weber-ampere characteristic, $\sum_{g}=\frac{1}{\sum R}$ - active conductivity and $L_{0}-$ leakage inductance NI EMT.

Considering that the voltage across the NI EMT is $u=U_{m} \operatorname{Cos} \omega t$ and taking into account the adopted approximation of the Weber-ampere characteristic of NI EMT, we write:

$$
\left\{\begin{array}{l}
i \sum c=-\frac{I_{c m}}{\Psi_{m}} \psi ; \\
i_{\Sigma g}= \pm \frac{I_{g m}}{\Psi_{m}} \sqrt{\Psi_{m}^{2}-\psi^{2}} \\
i_{\mu H}=a \psi+b \psi^{n},
\end{array}\right.
$$


From system (8) we obtain

$$
i=i_{\sum c}+i_{\sum g}+i_{\mu H}=\left(a-\frac{I_{c m}}{\Psi_{m}}\right) \psi+b \psi^{n} \pm \frac{I_{g m}}{\Psi_{m}} \sqrt{\Psi_{m}^{2}-\psi^{2}}(9)
$$

On the basis of (9) it is possible to construct a dynamic characteristic of NI EMT, which is identical with the dynamic loop of NI hysteresis (Fig. 2) [5,6].

The total active conductivity of the NI EMT voltage is determined by the dynamic coercive force $H_{c d}$ magnetic core material. If, induction $B=B_{m} \sin \omega t$ then,

$$
H_{c d}=H_{c}+0,125 \omega \sigma d^{2} B_{S} \sqrt{2 \varepsilon-1}
$$

where $B_{S}$ - saturation induction; $H_{c}$ - coercive force; $d$ magnetic material thickness; $\varepsilon$ - the conductivity of the magnetic material; $\sigma=\frac{B_{m}}{B_{s}}-$ core modulation ratio. With considering,

$$
\frac{U}{\sum R}=\frac{H_{c d} l}{w}=\frac{l}{w}\left(H_{c}+0,125 \omega \sigma d^{2} B_{S} \sqrt{2 \varepsilon-1}\right)
$$

Let's get the equivalent active resistance of NI EMT voltage.

$$
\begin{aligned}
& \sum R=\frac{U w}{l\left(H_{c}+0,125 \omega \sigma d^{2} B_{s} \sqrt{2 \varepsilon-1}\right)}= \\
& =\frac{\omega w^{2} S B}{l\left(H_{c}+0,125 \omega \sigma d^{2} B_{s} \sqrt{2 \varepsilon-1}\right)}
\end{aligned}
$$

From (8) we determine the equivalent parameters $\sum C$ and $L_{0}$ NI EMT.

$$
\begin{aligned}
& \sum C=\frac{a \Psi_{r}+b \Psi_{r}^{n}-\frac{1}{R} \sqrt{U_{m}^{2}-\Psi_{r}^{2} \omega^{2}}}{\omega^{2} \Psi_{r}}=(13) \\
& =\frac{a \psi_{r}+b \psi_{r}{ }^{n}-\frac{\omega}{R} \sqrt{\Psi_{m}^{2}-\Psi_{r}^{2}}}{\omega^{2} \Psi_{r}}
\end{aligned}
$$$$
L_{0}=\frac{\psi_{r}}{\frac{1}{\psi_{m}}\left(I_{c m} \psi_{r}+I_{g m} \sqrt{\psi_{m}^{2}-\psi_{r}^{2}}\right)-b \psi_{r}^{n}}
$$

Let's define an expression for instantaneous values of current and active power in NI EMT [8-13].

Considering that the flux linkage in the dynamic characteristic of NI EMT $\psi=\psi_{m} \sin \omega t$ and taking into account $i_{\mu H}=a \psi+b \psi^{n}$, then for the current in the ascending branch of the hysteresis loop the following expression takes place:

$$
i=I_{1 m} \sin (\omega t+\alpha)-\frac{b}{4} \Psi_{m}^{n} \sin 3 \omega t ;
$$

where:

$$
\begin{aligned}
& I_{1 m}=\sqrt{\left(a \Psi_{m}+\frac{3}{4} b \Psi_{m}^{n}-I_{c m}\right)^{2}+I_{g m}^{2}} ; \\
& \alpha=\operatorname{arctg} \frac{I_{g m}}{a \Psi_{m}+\frac{3}{4} b \Psi_{m}^{n}-I_{c m}} .
\end{aligned}
$$

If we assume that the instantaneous power in NI EMT is equal to:

$p=U_{m} I_{1 m} \cos \omega t \sin (\omega t+\alpha)-\frac{b}{4} U_{m} \Psi_{m}^{n} \cos \omega t \sin 3 \omega t ;$

then the active power taking into account (12), (13), (14) and (16) is determined from the expression:

$P=\frac{w^{2} S^{2} \omega^{2} B_{m}^{2}}{2} \cdot \frac{1}{\sum R}=2 \pi^{2} w^{2} S^{2} f^{2} \sigma B_{m}^{2}$

where $\sum \sigma=\frac{1}{\sum R}$ - specific electrical conductivity.

\section{Conclusion}

Thus, expressions (12), (13), and (14) determine the equivalent parameters $\sum g, \sum C$ and $L_{0}$ NI EMT is distinguished by a sufficiently high accuracy in comparison with the known calculation formulas.

The use of equivalent parameters of the generalized NI EMT model allows obtaining more accurate analytical expressions when calculating the equivalent circuit of ferroresonance with EMT and network capacities.

Expression (18) is distinguished by a sufficiently high accuracy in comparison with the known formulas for calculating the active power in the EMT and determines the losses in the NI EMT at ferroresonance in a wide range of frequency and effective voltage values.

\section{References}

1. Saenko Yu.L., Popov A.S. Investigation of ferroresonance processes taking into account the variation of the weber-ampere characteristics of voltage transformers // Technical Electrodynamics. -2012. - No. 6. - S. 51-57.

2. Hoshimov, F.A., Bakhadirov, I.I., Erejepov, M., Djumamuratov, B. (2019) Development of method for normalizing electricity consumption E3S Web Conf 139 doi:10.1051/e3sconf $/ 201913901074$

3. B.A. Abdullaev, A.A. Alimov, D.A. Xalmanov. To the problem of the calculation capacity of the nonlinear inductance // Seventh World Conference on Intelligent Systems for Industrial Autamation // - Tashkent .: 2012 -- p. 112-115.

4. Abdullaev B. Generalized models of nonlinear passive elements of electrical circuits and systems. $-\mathrm{T}$. Tashkent State Technical University, 2015. - S. 180.

5. Abdullaev B., Begmatov Sh.E. Application of a generalized model to the analysis of a secondary power supply. // Problems of energy and resource conservation. Tashkent, 2018, No. 3-4. S.150-153.

6. Sh.E.Begmatov. An innovative way to create secondary power sources with high energy performance.// Technical science and innovation. Tashkent, 2020, No. 2. Pages - P.120-125.

7. Begmatov Sh.E., Khalbutaeva Kh.I., Dusmukhamedova S.A. Study Of Ferroresonance In Electric Networks Taking Into Account The Generalized 
Model Of Nonlinear Inductance // World Conference. Science and life // Sofia .: 2020.- P.20-23.

8.Allayev, K.R., Fedorenko, G.M.,Postnikov, V.I.,Ostapchuk, L.B. Asynchronous generators as power system's natural dampers. 43rd International Conference on Large High Voltage Electric Systems 2010, CIGRE 20102010, 9p43rd International Conference on Large High Voltage Electric Systems 2010, CIGRE 2010; Paris; France; 22 August 2010.

9.Fazylov, Kh.F.,Allaev, K.R. Analysis of the operation of an electrical system during simultaneous operation of synchronous and asynchronous generators. Power engineering New York Volume 18, Issue 3, 1980, Pages 81-88.

10.Fazylov, Kh.F.,Allaev, K.R. Asynchronous turbogenerators with stator excitation and the prospects for their utilization. Power engineering New York Volume 23, Issue 2, 1985, Pages 7-13.

11.Fazylov, Kh.F.,Allaev, K.R. Calculation and experimental analysis of conditions of electrical power systems containing induction generators Power Engineering New York Volume 27, Issue 6, 1989, Pages 27-34.

12.Allaev K., Makhmudov T. Research of small oscillations of electrical power systems using the technology of embedding systems. Electrical Engineering, 2020; Issue 1: 309-319.DOI 10.1007/s00202-019-00876-9

13.Allaev K., Makhmudov T. Prospects of diversification and ensuring energy safety of Uzbekistan. E3S Web Conf., Volume 139, 2019, Rudenko International Conference "Methodological problems in reliability study of large energy systems" (RSES 2019).https://doi.org/10.1051/e3sconf/201913901002 